\title{
BUDD CHIARI SYNDROME: AN UNEXPECTED DIAGNOSIS IN A GASTROENTEROLOGY PRACTICE IN CALABAR, NIGERIA.
}

\author{
KOOFFREH-ADA M., CHUKWUDIKE E., KAJOGBOLA G., EGHWUBARE F., OKONKWO U., NGIM O.
}

(Received 4 February 2019; Revision Accepted 26 February 2019)

\begin{abstract}
Background: Vascular disorders of the liver are relatively rare in clinical practice. Budd Chiari syndrome (BCS) is a potentially life-threatening condition. It describes a group of disorders characterized by hepatic venous outflow obstruction at any level from the small hepatic veins up to the junction of the inferior vena cava and the right atrium. The prevalence of Budd Chiari Syndrome is greatly influenced by geographical differences. There is a dearth of data in Nigeria regarding this condition.

Case report: We report the case of a thirty seven year-old woman presenting with an eight year history of recurrent abdominal swelling, jaundice and progressive weight loss. The patient was promptly screened for viral hepatitis B and $\mathrm{C}$, which were both negative. The laboratory work-up revealed mild hepatic dysfunction and an abdominal ultrasound scan showed mild hepatomegaly and ascites. Abdominal Computed Tomography (CT) scan findings were consistent with Budd Chiari Syndrome, based on the findings of an extensive thrombus, involving the common iliac and hepatic veins, in addition, the inferior vena cava (IVC) and right atrium.

Conclusion: This case report highlights the importance of the knowledge of Budd Chiari Syndrome and the diagnostic approach. This condition should be considered whenever investigating the aetiology of chronic or acute liver disease in Gastroenterology practice in Nigeria.
\end{abstract}

KEYWORDS: Budd Chiari Syndrome, Thrombosis, Hepatic Venous Outflow Obstruction, Ascites.

\section{INTRODUCTION}

George Budd, a British internist, first described three cases of hepatic vein thrombosis due to abscessinduced phlebitis in 1845. Five decades after in 1899, Hans Chiari an Austrian pathologist first described the pathologic features of a liver with "obliterating endophlebitis of the hepatic veins" ${ }^{1}$. The term BCS is used to refer to a varied group of clinical conditions presenting with hepatic venous outflow obstruction, located at any level from the small hepatic veins to the junction of the inferior vena cava (IVC) with the right atrium ${ }^{2}, 3$. This excludes sinusoidal obstruction syndrome and right-sided heart failure complicated by hepatic outflow obstruction ${ }^{4}$.

Budd Chiari syndrome is rare and occurs in 1 per 100,000 in the general population ${ }^{1}$. In the Western hemisphere the estimated incidence of BCS is one in 2.5 million per person-year with thrombosis of the hepatic vein being the most common presentation ${ }^{4,3}$. In a retrospective study in China, the reported incidence and prevalence of BCS was approximately 0.88 /million per year and $7.69 /$ million, respectively ${ }^{2}$. In Africa, epidemiological data regarding BCS are rare. Varied underlying risk factors are responsible for the geographic differences in BCS, with poverty being implicated in Asian patients ${ }^{4}$. Pure idiopathic membranous obstruction of the IVC only (or combined IVC and hepatic vein obstruction) is a common finding in Asia and Africa, accounting for more than $40 \%$ of cases in this region ${ }^{3,4}$. While thrombosis of hepatic veins are predominant in the West with less common involvement of the IVC ${ }^{4}$. Oral contraceptive use and myeloproliferative disorders have been linked with BCS cases in the West ${ }^{4}$.

The exact nature of the hepatic venous obstruction further classifies BCS as being either primary or secondary ${ }^{4}$. If flow is occluded due to an endoluminal lesion, then it is classified as being primary BCS ${ }^{3,4}$. Whereas when flow is obstructed by either compression or invasion of a lesion outside the hepatic venous outflow track, it is regarded as being secondary BCS (malignant and cystic extrinsic obstruction) 3,4 . Thrombosis is the leading cause of primary BCS ${ }^{4}$. The disease has been regarded as being a rare hepatic manifestation of an underlying prothrombotic state ${ }^{4}$. In $75 \%$ of patients, a hematologic disorder or

Kooffreh-Ada M., Department of Internal Medicine, University of Calabar Teaching Hospital, Nigeria.

Chukwudike E., Department of Internal Medicine, University of Calabar Teaching Hospital, Nigeria.

Kajogbola G., Radiology Unit, Asi Ukpo Diagnostic Centre Calabar, Nigeria.

Eghwubare F., Department of Internal Medicine, University of Calabar Teaching Hospital, Nigeria.

Okonkwo U., Department of Internal Medicine, University of Calabar Teaching Hospital, Nigeria.

Ngim O., Department of Surgery, University of Calabar Teaching Hospital, Nigeria. 
prothrombotic condition has been identified as the predisposing factor to BCS ${ }^{5}$.

Hematologic conditions that have been linked with BCS, include; polycythemia vera which accounts for 10 to 40 $\%$ of cases, while essential thrombocytopenia and myelofibrosis are less prevalent causes ${ }^{6}$. Endogenous erythroid-colony formation may be seen in up to $87 \%$ of patients diagnosed to have idiopathic BCS, this infers that most BCS patients in whom the cause is unknown may have a background myeloproliferative condition ${ }^{6}$. Prothrombotic disorders associated with BCS include; paroxysmal nocturnal hemoglobinuria, antiphospholipid syndrome and inherited deficiencies of protein $\mathrm{C}$, protein $\mathrm{S}$, and antithrombin $\mathrm{III}^{6}$.

Tumors such as hepatocellular and renal cancers have been linked with BCS, while rare causes include; inflammatory bowel disease, aspergillosis and Behçet's syndrome among others ${ }^{6}$.

Regardless of the aetiology of hepatic venous outflow obstruction, a rapid increase in the hepatic sinusoidal pressure and portal hypertension arises, leading to venous congestion and ischemic damage to the surrounding sinusoidal hepatocytes ${ }^{5}$. prompt relief of hepatic sinusoidal pressure is not carried out through either therapeutic measures or the development of a venous collateral system, then nodular regeneration, fibrosis and subsequently cirrhosis may set in ${ }^{4}$.

The clinical presentation of BCS may be fulminant, acute, subacute or chronic ${ }^{3,4}$. Patients with the acute syndrome have a short duration of symptoms, intractable ascites, and hepatic necrosis without the formation of venous collaterals ${ }^{6}$. While the subacute form, which is the most common, has a more insidious onset; ascites is present and hepatic necrosis may be minimal, because the hepatic sinusoids have been decompressed by a portal and hepatic venous collateral circulation. In chronic BCS, patients present with the features and complications of liver cirrhosis ${ }^{6}$.

Imaging is vital is making the diagnosis of BCS. Doppler ultrasound is relatively cheap and safe with sensitivity and specificity rates greater than $80 \% 3$. Conventional ultrasound findings show an enlarged caudate lobe, hepatomegaly, poor visualization of the hepatic veins, a compressed IVC ${ }^{7}$. Intrahepatic collaterals, splenomegaly and the presence of ascites would suggest the subacute or chronic form of the disease 7 . The identification of collateral vessels with drainage into the subcapsular or intercostal veins is highly suggestive of BCS ${ }^{7}$ Computed tomography findings in acute BCS may include normal liver morphology, patchy enhancement, an enlarged caudate lobe, a compressed IVC, the absence of hepatic veins, and ascites ${ }^{7}$. Findings in subacute BCS depends on the type of venous involvement and may include the presence of portosystemic and intrahepatic collaterals as well as hepatic artery enlargement ${ }^{7}$. Typically, the IVC is compressed (suggesting secondary $\mathrm{BCS}$ ) or thrombosed (seen in primary BCS), in addition patients may present with portal vein thrombosis that is secondary to the profound flow disturbance ${ }^{7}$. Chronic BCS is associated with an alteration in the morphology of the liver and regenerative nodule formation ${ }^{7}$. These nodules characteristically show hyper-attenuation in the arterial phase and persists even in the portal phase, patchy enhancement may also be seen in this stage of the disease ${ }^{7}$.

The principles of managing BCS include; controlling the further progression of ascites with the use of diuretic agents, dietary salt restriction and paracentesis. In addition, the use of anticoagulants to prevent the progressive extension of the venous thrombosis as well as the treatment of an identifiable underlying cause ${ }^{2}$. Other therapies that have been employed include; thrombolytic therapy, angioplasty (percutaneous or transhepatic), transjugular intrahepatic portosystemic shunts and surgery (peritoneovenous shunts, liver transplant) ${ }^{2}$.

\section{CASE REPORT}

A thirty seven year old female trader sought medical care on account of recurrent abdominal swelling of 8 years duration (shortly following her last confinement). There was no leg swelling, cough, dyspnea, orthopnea, paroxysmal nocturnal dyspnea, facial swelling or oliguria. She had jaundice in the past and progressive weight loss. There was no history of blood transfusion, sharing of sharps and surgical procedures. She admitted the use of contraceptives (injectable), drank alcohol occasionally in the past but no use of tobacco in the past. Furthermore, no history of spontaneous abortions, gastrointestinal bleeding or thromboembolic phenomena. The physical examination revealed a chronically ill-looking woman with a markedly distended abdomen and distended superficial abdominal veins. There was hepatomegaly and marked ascites. Cardiovascular and respiratory examinations were unremarkable.

Abdominal ultrasound scan revealed a mildly enlarged liver with a normal parenchymal echogenicity. The hepatic vein was prominent, with a normal portal vein diameter $(1.2 \mathrm{~cm})$.

A contrast enhanced computed tomographic (CECT) scan demonstrated a slightly enlarged liver. No focal hepatic mass lesion was however seen. Delayed subtle hepatic parenchymal enhancement was also noted. Caudate lobe enlargement was also identified. There was an extensive isodense intra-luminal filling defect along the entire length of the IVC (from the right atrium to the common iliac veins), with hepatic veins, right renal vein and right atrial components. Normal intrahepatic biliary channels were seen. Copious ascitic fluid collection was also noted. The uterus, both adnexa and the urinary bladder were normal. The conclusion was that of an extensive common iliac, hepatic veins, IVC and right atrial thrombosis in keeping with BCS. See images of CT scan below. 
Coronal and axial contrast enhanced computed tomographic images
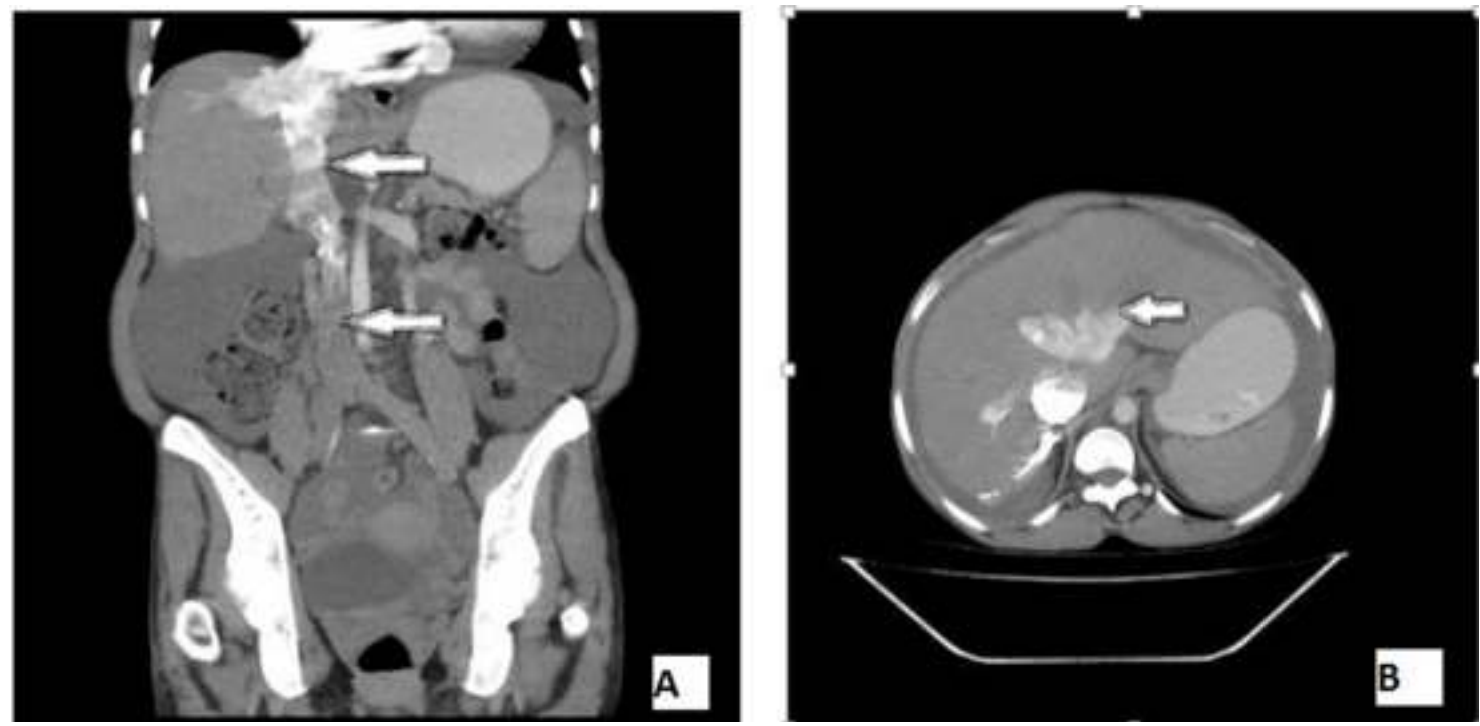

Figure 1:Coronal (A) and axial (B) contrast enhanced computed tomographic images showing extensive isodense intra-Iuminal partial filling defects (white arrows) within the suprarenal inferior vena cava (IVC) and the hepatic veins extending inferiorly into the common iliac veins.
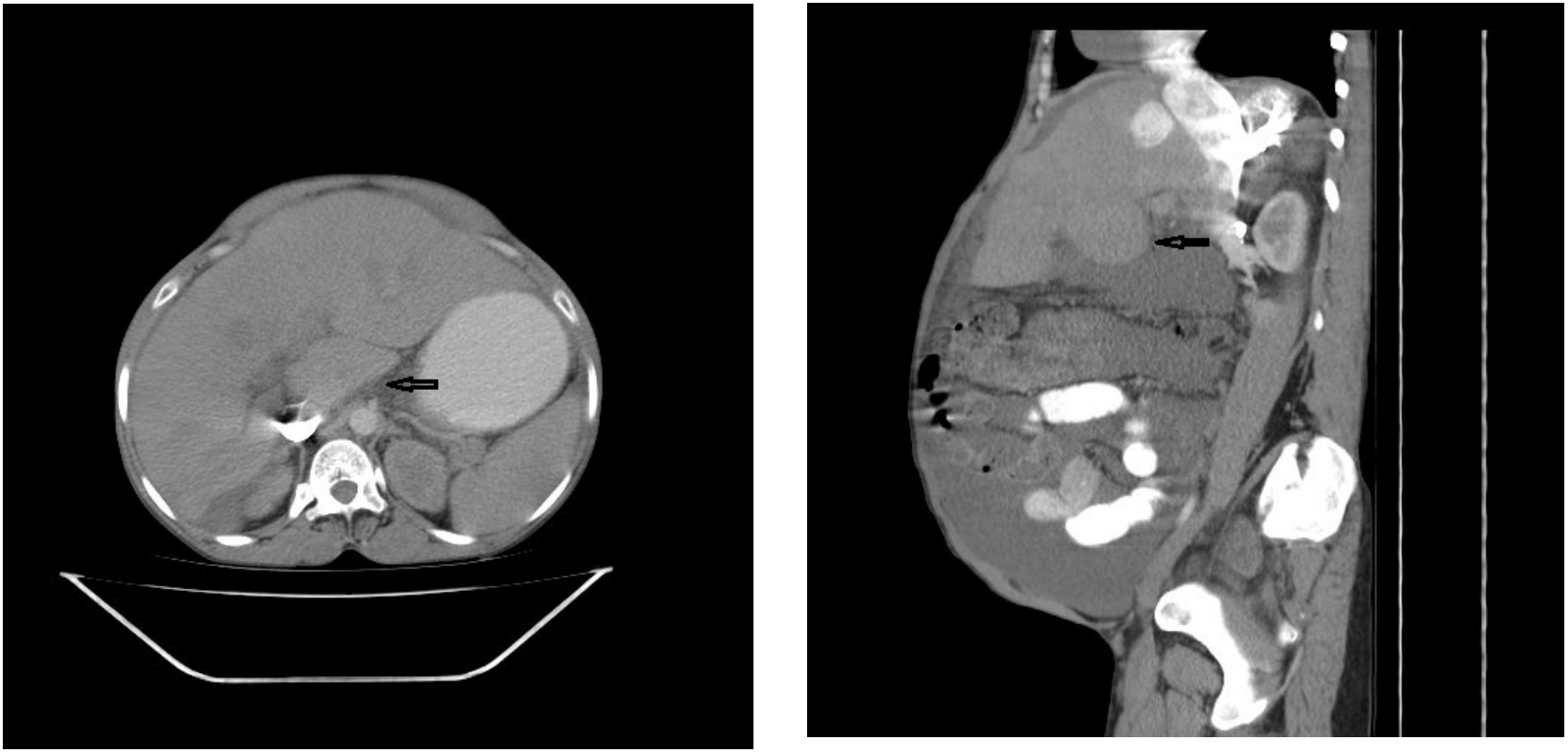

Figure 2: Axial contrast enhanced computed tomographic image showing caudate lobe enlargement.

Routine blood tests (See table below) were carried out, it revealed normal liver enzymes, hypoalbuminaemia and high serum to ascitic albumin gradient (SAAG). Hematologic tests to further investigate a possible hypercoagulable state could not be carried out due to lack of funds. Lactulose and diuretics (spironolactone and frusemide) were subsequently commenced. In addition, therapeutic paracentesis with salt poor albumin (three sessions) was also done. In view of the diagnosis of BCS she was placed on anticoagulation therapy (low molecular weight heparin and Dabigatran) with target INR of 2-3. Currently, she's stable evident by the resolution of ascites and her improved overall wellbeing. She is currently being seen regularly on an out-patient basis. 
Table showing summary of laboratory investigations

\begin{tabular}{|c|c|c|}
\hline Investigations & Results & Reference value \\
\hline \multicolumn{3}{|l|}{ Liver function test } \\
\hline ALT U/L & 19 & $0-40$ \\
\hline AST U/L & 25 & $0-40$ \\
\hline Total Bilirubin mg/dl & 34.6 & $2-17$ \\
\hline Conjugated bilirubin $\mathrm{mg} / \mathrm{dl}$ & 26.2 & $2-7$ \\
\hline Alkaline phosphatase U/L & 30 & $22-97$ \\
\hline Total protein $\mathrm{g} / \mathrm{dl}$ & 77 & $62-82$ \\
\hline Albumin $\mathrm{g} / \mathrm{dl}$ & 33 & $36-52$ \\
\hline \multicolumn{3}{|l|}{ Urea/Electrolyte } \\
\hline Urea umol/L & 5.37 & $2.2-7.2$ \\
\hline Creatinine umol/l & 105.2 & $44.2-106.1$ \\
\hline Potassium mmol/l & 4.5 & $3.5-5.1$ \\
\hline Chloride $\mathrm{mmol} / \mathrm{l}$ & 92.1 & $98-107$ \\
\hline Bicarbonate $\mathrm{mmol} / \mathrm{l}$ & 32.4 & $\begin{array}{l}23-29 \\
38-48\end{array}$ \\
\hline \multicolumn{3}{|l|}{ Full blood count } \\
\hline PCV\% & 29 & $38-48$ \\
\hline Haemoglobin g/dl & 9.6 & $11.5-15.5$ \\
\hline MCV um ${ }^{3}$ & 100 & $80-100$ \\
\hline $\mathrm{MCH}$ pg & 33.1 & $27-32$ \\
\hline $\mathrm{MCHC} \mathrm{g} / \mathrm{dl}$ & 33.1 & $3-35$ \\
\hline Platelet $\times 10 / \mathrm{mm}$ & 149 & $150-450$ \\
\hline WBC count(total) $\times 10 / \mathrm{mm}$ & 4.1 & $4-10$ \\
\hline Neutrophils $\%$ & 56.9 & $45-70$ \\
\hline Lymphocytes \% & 24.9 & $2-45$ \\
\hline Monocytes \% & 11.3 & $0-10$ \\
\hline Eosinophils \% & 6.7 & $0-5$ \\
\hline Basophils \% & 0.2 & $0-2$ \\
\hline Erythrocyte & 75 & $0-20$ \\
\hline $\begin{array}{l}\text { sedimentation } \\
\mathrm{mm} / \mathrm{hr}\end{array}$ & & \\
\hline INR & 1.06 & $<1.1$ \\
\hline APTT seconds & 41.0 & $30-40$ \\
\hline \multicolumn{3}{|l|}{ Tumor markers } \\
\hline Alpha-fetoprotein $\mathrm{ng} / \mathrm{ml}$ & 6.3 & $0-10$ \\
\hline $\mathrm{CA} 125 \mathrm{U} / \mathrm{ml}$ & 29 & $0-35$ \\
\hline HBsAg & Negative & \\
\hline Anti-HCV & Negative & \\
\hline HIV screening & Negative & \\
\hline \multicolumn{3}{|l|}{ Ascitic fluid analysis } \\
\hline Leucocytes & Negative & \\
\hline AFB & None seen & \\
\hline $\begin{array}{l}\text { Serum ascitic albumin } \\
\text { aradient (SAAG) }\end{array}$ & $>1.1$ & \\
\hline
\end{tabular}

\section{DISCUSSION}

Reports of veno-occlusive disorders of the liver are rare in Nigeria, probably due to either misdiagnosis or a low index of suspicion. The earliest report of BCS was over four decades ago by Edington et al in north central Nigeria in a young child who had a liver histology report which revealed chronic venous congestion that was consistent with hepatic venous outflow obstruction ${ }^{8}$. In western countries, women in the third or fourth decade of life were found to be more at risk of developing BCS 9. This observation was also seen in the BCS cases reported in Brazil and Iran 9, 10. A similar finding was found in our index patient who was aged thirty seven years.

The use of contraceptives has been identified in up to $50 \%$ of BCS cases in females ${ }^{9}$. The relative risk of the developing BCS following contraceptives use is up to $2.4 \%$. Pregnancy and the puerperium are also considered predisposing factors ${ }^{9}$. Our patient admitted to using injectable contraceptives in the past. However, in a case reported by Mahdavi et al, of a female BCS patient who had a history of use of injectable contraceptives they were unable to find a link between BCS and injectable contraceptives. They rather postulated the role of the idiopathic form of BCS and occult myeloproliferative disorders ${ }^{10}$. Obstruction of a single hepatic vein is generally not evident clinically. However, blockage of two or more major hepatic veins increases the sinusoidal pressure and reduces sinusoidal blood flow ${ }^{1}$. The subsequent, massive 
hepatocellular damage could result in a fulminant (acute) course of the disease with patients presenting with vomiting, and mild jaundice 1 . The finding of hepatosplenomegaly, distended superficial abdominal veins and ascites in our patient suggest the chronic form of $\mathrm{BCS}^{1,3}$.

Considering that viral hepatitis B is the leading cause of chronic liver disease in our environment ${ }^{11}$. Preliminary screening for hepatitis $B$ and $C$ viral infection were however negative. Further laboratory findings of a relatively normal liver enzymes, reduction in serum albumin and high SAAG were consistent with findings seen in chronic forms of BCS ${ }^{2}$.

Doppler ultrasonography is a useful first line investigation, while computed tomography (CT) scan and magnetic resonance imaging (MRI) are second line tools ${ }^{3}$. A combination of both doppler ultrasonography and either MRI or CT imaging should suffice to diagnose most cases of BCS ${ }^{3}$.

The CT scan finding in our patient revealed an intraluminal filling defect along the entire length of the inferior vena cava, indicating obstruction (from either thrombosis or phlebitis) occurring within the vessel, this would suggest she had primary BCS . The CT scan excluded the presence of a tumor, abscess, or a cyst originating outside the veins that may be causing an obstruction to the hepatic outflow via compression or invasion (i.e. secondary BCS). Results of tumor markers for liver and ovarian cancer were within normal limits.

Despite the identification of some risk factors in our patient, a major setback in establishing an underlying cause of BCS was hampered, due to lack of funds. Equally, some of the prothrombotic screeningtests are unavailable in our environment.

Once a definitive diagnosis of BCS is made, indefinite anticoagulation is necessary as a first line measure to prevent progression of venous thrombosis ${ }^{2,12}$. Based on this recommendation, low molecular weight heparin and dabigatran was commenced in our patient. Ascites is managed by restricting the patient's sodium intake to $90 \mathrm{mmol}$ per day and administering spironolactone and furosemide ${ }^{2}$. Severe ascitic fluid can be mobilized by carrying out large-volume paracentesis and intravenous infusions of albumin ${ }^{2}$. Studies have shown that medical treatment alone may be satisfactory with a promising prognosis ${ }^{2}$. We were able to achieve the above medical strategies for our patient with a favorable outcome (based on the steady resolution of ascites and her improved overall wellbeing).

Transjugular intrahepatic portosystemic shunts may be useful especially when vessel clearing by fibrinolysis or angioplasty, or anticoagulation have failed, while liver transplantation is indicated in refractory or fulminant cases 1,2 . However, these modalities of treatment are currently unavailable in our environment

\section{CONCLUSION}

Although the diagnosis of BCS is rare in our clinical practice, it is expected that with advanced imaging techniques the diagnosis of this condition may ultimately improve in our environment. This case report therefore, highlights the need for Gastroenterologists in Nigeria to have a high index of suspicion in patients who present to their practice with the clinical features of liver disease in which the diagnosis is clearly not elucidated. This case report also showed that a major hindrance in evaluating a suspected case of BCS in our environment would be the unavailability of some of the hematologic tests required to screen BCS and lack of finances in carrying out the tests where available.

\section{REFERENCE}

Martens P, Nevens F.2015. Budd-Chiari Syndrome. United European Gastroenterology Journal. 3(6) 489-500.

Stevens WE, P Abhitabh. Vascular Diseases of the Liver. In: Feldman M, Friedman LS, Brandt LJ (eds). Sleisenger and Fordtran's Gastrointestinal and Liver disease: Pathophysiology, diagnosis, management. Vol. II, 9th ed. Philadelphia: Elsevier Saunders, 2010: 83:1371-74.

Aydinli M, Bayraktar Y. 2007. Budd-Chiari syndrome: Etiology, pathogenesis and diagnosis. World $\mathrm{J}$ Gastroenterol.13(19) 2693-2696.

Lin M, Zhang F, Wang Y, Zhang B, Zhang W, Zou X. 2017. Liver cirrhosis caused by chronic BuddChiari syndrome. Medicine.96:34.

Cura, M, Haskal Z, Lopera J. Diagnostic and Interventional Radiology for Budd-Chiari Syndrome. 2009. www.rsna.org/rsnarights. Site assessed 23rd January 2019.

Menon KV, Shah V, Kamath PS.2014. The Budd-Chiari Syndrome. N Engl J Med.350:578-85.

Ferral H, Behrens, Lopera J. 2012. Budd Chiari Syndrome. AJR.199:737-745.

Edington G.M, Haggie M.K. 1977. Hepatic vein occlusion disease: Report of a case in the North Central state of Nigeria. Nigerian Journal of Pediatrics. 4 (1): 6.

Falcão CK, Fagundes GF, Lamos GC, Felipe-Silva A, Lovisolo SM, Martines JA.2015. Budd-Chiari Syndrome: an unnoticed diagnosis. Autopsy and Case Reports.5(2):17-25.

Mahdavi A, Hosseini Jadda SH H. 2012. Occurrence of Budd-Chiari Syndrome as Adverse Effect of Long Term DMPA Injections: A Case Report and Review the Literatures. Journal of Family and Reproductive Health.6(4).

Kooffreh-Ada M, Okpara H, Oku A, Okonkwo UC, Ihekwaba A. 2015. Risk factors of chronic liver disease amongst patients receiving care in a Gastroenterology practice in Calabar: IOSR Journal of Dental and Medical Sciences.14 (12) 06-13.

Mancuso A.2014. An update on management of Budd Chiari Syndrome. Annals of Hepatology. 13 (3): 323-326. 
